\title{
Corticosteroids in Duchenne Muscular Dystrophy-A Deflazacort Review
}

\author{
Jordan S Dubow and James M Meyer \\ Marathon Pharmaceuticals, LLC, Northbrook, IL, US
}

DOl: http://doi.org/10.17925/USN.2016.12.01.12

\begin{abstract}
Duchenne Muscular dystrophy (DMD) is a devastating disorder that is the most common and severe form of childhood onset muscular dystrophy. While guidelines exist that promote a multidisciplinary approach to treatment of DMD, surveys have identified inconsistent adherence to the recommendations. This is particularly highlighted by the variability in the prescriptive habits of corticosteroids, which have a long history of literature supporting their use in DMD. Relatively recent publications have uncovered differences between countries in the prescription patterns of steroid regimens. Currently, treatment recommendations lack comparative data on the efficacy and safety of alternative dosing regimens of corticosteroids. New research is being conducted to help understand the benefits and risks of the most commonly used glucocorticoid regimens in DMD. Deflazacort is a glucocorticoid corticosteroid that is currently seeking approval in the U.S. This article summarizes the relevant published literature on deflazacort to help inform DMD-treating physicians on its impact on the disease.
\end{abstract}

\section{Keywords}

Duchenne muscular dystrophy, DMD, corticosteroids, deflazacort, review

Disclosure: Jordan Dubow and James Meyer are both employees of Marathon Pharmaceuticals, LLC.

open Access: This article is published under the Creative Commons Attribution Noncommercial License, which permits any noncommercial use, distribution, adaptation, and reproduction provided the original author(s) and source are given appropriate credit.

Received: January 25, 2016 Accepted: February 16, 2016 Citation: US Neurology, 2016;12(1):12-6

Correspondence: Jordan Dubow, MD, Marathon Pharmaceuticals, LLC, 1033 Skokie Blvd Suite 600, Northbrook, IL 60062, US. E: jdubow@marathonpharma.com

Support: The publication of this article was supported by Marathon Pharmaceuticals, LLC. The views and opinions expressed are those of the authors and do not necessarily reflect those of Marathon Pharmaceuticals, LLC.

Duchenne muscular dystrophy (DMD) is an X-linked disorder affecting one in 5,000 live male births which makes it the most common and most severe form of muscular dystrophy. ${ }^{1}$ The absence of the protein dystrophin leads to symptom onset typically between the ages of two to five years, with abnormal gait and frequent falls being hallmark signs. ${ }^{2,3}$ Other signs may include delays in motor milestones such as sitting, standing independently, climbing and walking, as well as delays in cognitive development. Untreated, patients with DMD will lose ambulation and become wheelchair dependent at a mean age of 9.5 years. ${ }^{4}$ In the second decade of life, complications of respiratory, cardiac, and orthopedic origin are common, with death typically occurring in the second or third decade due to respiratory failure and cardiomyopathy. ${ }^{5}$

Glucocorticoid corticosteroids are the only treatment currently recommended in guidelines to be effective in treating the signs and symptoms of DMD. ${ }^{6-8}$ In the ambulatory phase of the disease, corticosteroid treatment is aimed at preserving ambulation and minimizing future respiratory, cardiac, and orthopedic complications. However, they do not treat or correct the underlying genetic cause of DMD. In the nonambulatory phase of DMD, corticosteroids may decrease the chance of developing scoliosis, improve upper limb strength, and help maintain respiratory and cardiac function. Corticosteroids are often used by DMD patients over the long-term so monitoring and managing potential adverse event risks and complications is critical as the use of corticosteroids comes with known side effects. These may include weight gain, changes in behavior, short stature, delayed puberty, and others.

The practice parameters for DMD, published in 2010 were designed for the purpose of optimizing care for patients with DMD, focusing on a multidisciplinary approach to ensure that comprehensive care is provided across all clinics that treat neuromuscular disorders. ${ }^{6,7}$ Areas covered in the guidance include: management with corticosteroids, diagnostics, rehabilitation management, orthopedics, psychosocial management, cardiac, pulmonary, gastrointestinal (GI)/speech/swallowing/nutrition. It has been through the efforts of patient advocacy groups, professional organizations, and alliances that have led to the creation of resources to help inform and empower parents and patients to promote the implementation of comprehensive care internationally. ${ }^{9}$

While this comprehensive multidisciplinary care approach has been demonstrated as the best model of care for people living with $\mathrm{DMD}$, the implementation of this model may not be possible in all areas, and evidence has been published that compliance to the published care standards is not universal. ${ }^{10}$ This may be due to the fact that many DMD patients will only visit a team of specialists on an annual basis and will typically rely on local physicians more regularly throughout the year. One 
aspect where this is evident is with the underutilization of corticosteroids in DMD patients despite years of data demonstrating their benefits.

While data from randomized controlled trials have shown benefit of corticosteroids on muscle strength and function, and data from nonrandomized trials have suggested longitudinal benefit, there is not a consensus on the most appropriate steroid regimen. Currently, treatment recommendations lack comparative data on the efficacy of alternative dosing regimens. Relatively recent publications have uncovered differences between countries in the prescription patterns of steroids regimens. The Finding the Optimum Regimen for Duchenne Muscular Dystrophy (FOR DMD) trial is aimed at helping to understand the benefits and risks of the most commonly used glucocorticoid regimens in DMD. ${ }^{11}$ In the US, the most common glucocorticoid regimens are either prednisone $0.75 \mathrm{mg} / \mathrm{kg} /$ day, which is not approved by the FDA for the treatment of DMD or deflazacort $0.9 \mathrm{mg} / \mathrm{kg} /$ day imported from outside of the United States. Deflazacort has been used for the treatment of DMD for over 20 years and published data demonstrates it to be a potentially safer and more effective alternative to prednisone. Deflazacort appears to have a positive impact on multiple aspects of the disease and could be used across the entire DMD population regardless of underlying genetic mutation. Efforts to achieve approval for deflazacort from the FDA are close to being submitted for review. As deflazacort may be approved for DMD and available to patients in the US in early 2017, this article summarizes the published literature on deflazacort to help inform DMDtreating physicians on its impact on the disease.

\section{Muscle Strength/Motor Function/Loss of Ambulation}

Numerous publications have demonstrated the benefits of deflazacort on muscle strength and function. In a cohort of 54 boys with DMD, where 30 received active treatment and 24 were untreated, Biggar et al., 200112 reported that over a 6-year period patients treated with deflazacort were able to climb a flight of stairs to a later age $(10.9 \pm 1.9$ years versus $9.2 \pm 1.1$ years, $p<0.001)$ and rise from a floor to a later age $(10.7 \pm 1.9$ years versus $8.8 \pm 1.4$ years, $p<0.001$ ) than untreated boys. Over the six-year period, all of the patients in the non-treatment group lost ambulation, while only seven (23\%) in deflazacort treatment group did so and at a significantly later age $(12.3 \pm 2.7$ years versus $9.8 \pm 1.8$ years, $p<0.05)$. There was also a dramatic increase in the proportion of patients older than 10 years of age who could still walk in the treatment group compared to the non-treatment group (70\% versus 0\%, p<0.0001). Using the same cohort Alman et al., $2004^{13}$ described similar results over a longer timeframe, reporting that over a five- to eight-year period patients in the active deflazacort treatment group were able to climb a flight of stairs longer than untreated patients (10.9 \pm 1.9 years versus $9.2 \pm 1.0$ years). Patients in the active deflazacort treatment group remained ambulatory for longer than untreated patients $(12.3 \pm 2.7$ years versus $9.8 \pm 1.8$ years $)$.

Another paper by Biggar et al., $2006^{14}$ reported on the benefits of deflazacort treatment in 40 boys in their second decade compared to data from 34 boys who were not treated with deflazacort, over a 14 year period (January 1990 until December 2004). Seventy percent (28) of the treated boys (40) could rise from the floor to standing at 10 years of age, $48 \%$ at 12 years of age, whereas, $17 \%$ of the untreated boys (34) could rise from the floor at 10 years of age, and none at 12 years of age. Seventy percent of the treated boys could climb four stairs at 10 years of age and $55 \%$ at 12 years of age, whereas, only $6 \%$ of untreated boys could climb four stairs at 10 years of age and none at 12 years of age. All treated boys could walk $10 \mathrm{~m}$ at 10 years of age and $81 \%$ at 12 years of age, whereas, all 34 untreated boys stopped walking by 12 years of age.

In a retrospective analysis, Schara $2001^{15}$ analyzed the outcome of longterm steroid therapy for DMD using results with an average follow up period of 65 months. Nineteen male patients receiving deflazacort 0.9 $\mathrm{mg} / \mathrm{kg}$ daily were compared with the natural history of DMD available in the literature. Additionally, 13 of the 19 male patients were compared with an age-matched control group of 13 patients with DMD who were not receiving steroid treatment. The results suggested diminished loss of muscle strength and function in deflazacort treated patients with DMD compared to the natural history and this was observed to be more apparent with increasing age (in patients who started therapy early i.e. five years of age). The statistical analysis of 13 deflazacort treated and 13 age-matched untreated patients showed that the average muscle strength was significantly greater in the treated group $(p<0.0001)$. All deflazacort treated patients were able to walk independently throughout the study up to a mean age of 13 years, as compared to 9.3 years in natural history.

A randomized, double-blind study of two doses of deflazacort $(0.9 \mathrm{mg} / \mathrm{kg} /$ day and $1.2 \mathrm{mg} / \mathrm{kg} /$ day) compared to prednisone $0.9 \mathrm{mg} / \mathrm{kg} /$ day and placebo over 12 weeks demonstrated that both doses of deflazacort and prednisone significantly improved muscle strength compared to placebo (strength increased in all active treatments and decreased in the placebo group). ${ }^{16}$ However, after 12 months weight gain was significantly greater with prednisone compared to placebo whereas the deflazacort group was not different than placebo. Bonifati et al., 200017 examined the effect of deflazacort versus prednisone treatment on muscular and motor function in 18 boys whose age ranged from 5.2 to 14.6 years (mean, 7.3 years) with DMD in a double-blind, randomized trial over a one-year period. Patients were treated with either deflazacort $(0.9 \mathrm{mg} / \mathrm{kg} /$ day $)$ or prednisone $(0.75 \mathrm{mg} / \mathrm{kg} /$ day) on the basis of age and functional score at the onset of treatment. The authors evaluated four limb muscles with the MRC scale and the performance of four functions (walking, climbing stairs, Gowers' maneuver, and rising from a chair). At 12 months, the effect of both steroids was examined by comparing the status of the treated patients with another group of untreated DMD patients that served as natural history control. Results demonstrated that both steroids were equally effective in improving motor function and functional performances. After 12 months of treatment the prednisone group gained significantly more weight than the deflazacort group (5.08 kg vs $2.17 \mathrm{~kg}$; $p<0.05$ ).

Balaban et al., $2005^{18}$ examined the long-term effect of deflazacort treatment on motor, spinal, and pulmonary function in 49 boys with DMD over a seven-year period, where 18 patients received prednisone, 12 received deflazacort, and 19 received no steroid. In a battery of motor function tests, patients treated with either deflazacort or prednisone lost their ability to walk, get up, climb, stand, lift weights, and lift their hand at a significantly later age than untreated patients ( $p<0.05$ in all cases), and there was no significant difference in outcome between the two treatments ( $p=0.393$ to 0.967 ). Similar results were seen in measurements of hand strength, with deflazacort appearing to perform somewhat better than prednisone within the small sample size employed. 
Angelini et al., ${ }^{19}$, in a double-blind, placebo controlled trial in 28 patients with DMD randomized in a 2:1 fashion to deflazacort $2 \mathrm{mg} / \mathrm{kg} / \mathrm{QOD}$ or placebo over two years, demonstrated significant benefits of deflazacort on motor strength and motor function. After six months of treatment, the deflazacort group showed significant improvement in climbing stairs $(p<0.01)$, Gower's maneuver and walking $(p<0.0025)$ compared to placebo. After one year of treatment all of the above changes remained significant and muscle strength was significantly better in the treatment group $(p<0.05)$. After two years of treatment, deflazacort-treated boys had significant improvements in muscle strength, walking chair rising and Gower's maneuver compared to placebo. The mean time to loss of ambulation for the deflazacort-treated group after starting the trial was 20.5 months compared to 33.2 months for the placebo group $(p<0.05)$. Deflazacort-treated patients lost ambulation at a median age of 11.8 years compared to 10.5 years in the placebo group.

Houde et al., $2008^{20}$ reported on a study which examined the long-term effects of deflazacort treatment in 79 boys with DMD, in which 37 received active treatment and 42 were untreated. Data were collected over an eight-year period, with the mean length of treatment reported to be 66 months. The results of this study demonstrated that boys who received long-term treatment with deflazacort stopped walking at $11.5+$ /- 1.9 years, compared with $9.6+/-1.4$ years for untreated boys.

A further paper was published by Biggar et al., in $2004^{21}$ which compared two protocols for using deflazacort in the treatment of $\mathrm{DMD}$; the lowerdose 'Naples protocol' (0.6 mg/kg/day, 20 days on and 10 days off, vitamin $\mathrm{D}$ and calcium daily if osteoporosis present) and the higher-dose 'Toronto protocol' $(0.9 \mathrm{mg} / \mathrm{kg} /$ day, daily, vitamin D and calcium daily). A total of 37 and 32 boys with DMD between the ages of 8 and 15 years were treated with deflazacort for at least four years using the Naples and Toronto protocols, respectively, then compared with non-treatment groups containing 19 and 30 patients, respectively. Deflazacort treatment started between four and eight years of age. The results were compared with age-matched controls in the two groups (19 for the Naples protocol and 30 for the Toronto protocol). For the boys treated with Naples protocol, $97 \%$ were ambulatory at nine years (control, 22\%), 35\% at 12 years (control, 0\%), 25\% at 15 years (control, 0\%). For the 32 boys treated with the Toronto protocol, $100 \%$ were ambulatory at nine years (control, $48 \%$ ), $83 \%$ at 12 years (control, $0 \%$ ) and $77 \%$ at 15 years (control, $0 \%$ ). No aids or leg braces were used for ambulation.

Wang et al., 2014²2 examined the effects of corticosteroids in delaying age at full-time wheelchair use in DMD, using registry data from 1,057 males from Duchenneconnect. In this registry, deflazacort was used by approximately $58 \%$ of those taking steroids and was noted to prolong ambulation to a median of 14 years compared to prednisone, which delayed wheelchair free survival to a median of 13 years $(p=0.0013$ between deflazacort and prednisone). Similarly, Bello $2015^{23}$ reported the results from the Cooperative International Neuromuscular Research Group (CINRG) which prospectively observed 340 DMD patients aged two to 28 years who received treatment with different corticosteroid regimens (involving both deflazacort and prednisolone). Prednisolone was administered while ambulatory to 150 participants (54.1\% of treated) and deflazacort to 91 (32.9\%), with 36 participants (13\%) switching drugs while ambulatory. Deflazacort was associated with later loss of ambulation than prednisolone (median loss of ambulation with deflazacort at 13.9 years compared to median loss of ambulation with prednisone of 11.2 years; $p=0.0001$; lower hazard ratio $0.294 \pm 0.053$ vs $0.490 \pm 0.08, p=0.003$ ).

\section{Scoliosis}

Untreated boys with DMD develop significant scoliosis, often requiring surgery. Following surgery, boys typically lose the ability to feed themselves. Deflazacort has proven to reduce the risk of scoliosis. Using the same cohort of 54 boys with DMD, where 30 received deflazacort and 24 were untreated, Lebel et al., $2013^{24}$ and Alman $2004^{13}$ demonstrated the benefits of deflazacort on reducing the development of scoliosis. Their data demonstrated that between Years 5 and 8, 5 patients (17\%) in the active deflazacort treatment group had developed scoliosis (spinal curve $>20^{\circ}$ ) and underwent surgery, compared to $16(67 \%)$ in the non-treatment group, 15 (63\%) of which also underwent surgery $(p<0.001)$. Subsequently, after a 15 -year period, only six patients (20\%) in the active deflazacort treatment group had developed scoliosis and underwent surgery, compared to 22 (92\%) in the non-treatment group. Similarly, patients receiving deflazacort treatment exhibited reduced spinal curve measurements versus those with no treatment, $11^{\circ}(95 \%$ confidence interval $[\mathrm{Cl}], 2^{\circ}$ to $\left.21^{\circ}\right)$ to $32^{\circ}\left(95 \% \mathrm{Cl}, 21^{\circ}\right.$ to $\left.43^{\circ}\right)$. By the end of the study, $78 \%$ (95\% confidence interval, $57 \%$ to $89 \%$ ) in the treatment group had avoided surgery for scoliosis entirely compared to $8.3 \%$ ( $95 \% \mathrm{Cl}, 0.8 \%$ to $28 \%$ ) in the non-treatment group ( $p=5.8 \times 10-7, \log$-rank and chi-square tests).

Houde et al., $2008^{20}$ demonstrated that scoliosis was much less severe in deflazacort treated (14 +/- 2.5 degrees) vs. untreated boys ( $46+/-24$ degrees), with no spinal surgery necessary in treated boys $(0 \%$ versus $43 \%$ ). Similarly, Biggar $2006^{14}$ showed that by 18 years of age, 30 of 34 (90\%) of the untreated boys developed scoliosis with spinal curve $>20 \%$ compared to only 4 of 40 (10\%) boys treated with deflazacort.

Balaban et al., $2005^{18}$ examined the long-term effect of deflazacort treatment on motor, spinal, and pulmonary function in 49 boys with DMD over a sevenyear period, where 18 patients received prednisone, 12 received deflazacort, and 19 received neither steroid. Spinal surgery for scoliosis was performed on 10 patients (53\%) in the non-treatment group compared to 2 patients (11\%) in the prednisone group and 0 patients in the deflazacort group; a result that was significant between treated and untreated populations $(p<0.05)$ but not between the two treatment options $(p>0.05)$.

\section{Cardiac Function}

Cardiomyopathy and cardiac dysfunction are major causes of morbidity and mortality in DMD patients. There is some evidence demonstrating benefits of deflazacort on cardiac disease in DMD patients. Mavrogeni et al., $2009^{25}$ examined the myocardium and sternocleidomastoid muscles in patients with DMD using imaging data from magnetic resonance imaging (MRI) and T2 relaxation time mapping. A total of 17 patients who had been treated with deflazacort for a minimum of seven years were compared with a second 17 who had not received any steroid therapy. While the sample size was very small and the MRI measurements were not taken across time, it was found that the cardiac and respiratory musculature of patients who had received deflazacort treatment was better preserved than that of untreated individuals, as reflected by higher $\mathrm{T} 2$ relaxation times. It was concluded that DMD patients on deflazacort are characterized by better preservation of the T2 relaxation time of myocardium and sternocleidomastoid muscles and better left ventricle (LV) systolic function. 
In the study of 79 DMD boys in which 37 were treated with deflazacort and 42 were not over an eight-year period, Houde et al., 200820 demonstrated that cardiac function was better preserved with the use of deflazacort. The study found a normal shortening fraction in treated $(30.8+/-4.5 \%)$ vs untreated boys $(26.6+/-5.7 \%, \mathrm{p}<0.05)$, a higher ejection fraction ( $52.9+/-6.3 \%$ treated vs $46+/-10 \%$ untreated, $p<0.05)$, and lower frequency of dilated cardiomyopathy (32\% treated vs $58 \%$ untreated, $\mathrm{p}<0.05)$ in the deflazacort-treated group.

Silversides et al., $2003^{26}$ examined the long-term effect of deflazacort treatment on myocardial function in 33 boys with DMD over a three-year period or longer, where 21 received active treatment and 12 were untreated. Despite the small sample size, instances of significant preservation in cardiac function were observed in the treatment group compared to the non-treatment group, including higher fractional shortening ( $33 \pm 7 \%$ versus $21 \pm 8 \%, p=0.002$ ), reduced left ventricular ejection fractions ( $5 \%$ versus $58 \%, p=0.001$ ), and smaller left ventricular end-systolic dimensions ( $30 \pm 6 \mathrm{~mm}$ versus $37 \pm 8 \mathrm{~mm}, \mathrm{p}=0.02$ ).

\section{Pulmonary Function}

As boys with DMD age, pulmonary function diminishes. Pulmonary support has made a vast improvement in prolonging survival in DMD. There are no approved drugs to treat the pulmonary aspect of the disease. However, many studies support the benefits of deflazacort on pulmonary function in DMD. Using the same cohort of 54 boys with DMD, where 30 received active treatment and 24 were untreated, Biggar et al., $2001^{12}$ (providing data over a six-year period) and Alman et al., 2004 ${ }^{13}$ (reporting interim results over a five- to eight-year period) found patients within the deflazacort treatment group had improved pulmonary function. Alman et al., $2004^{13}$ showed percent predicted forced vital capacity (FVC) of $72 \pm$ $11 \%$ for treatment group versus $35 \pm 10 \%$ for non-treatment. Biggar $2001^{12}$ demonstrated a significantly higher percent predicted FVC at age 13 (82.5 $\pm 14 \%$ versus $43.2 \pm 9 \%, p<0.002)$ and age $15(88 \pm 18 \%$ versus $39 \pm 20 \%$, $\mathrm{p}<0.001)$. Similarly, Silversides $2003^{16}$ found that patients on deflazacort exhibited improved pulmonary function in comparisons of FVC $(2.0 \pm 0.4 \mathrm{~L}$ versus $1.4 \pm 0.5 \mathrm{~L}, \mathrm{p}=0.001)$ and percent predicted FVC ( $83 \pm 12 \%$ versus $41 \pm 19 \%, p<0.001$ ) compared to untreated boys.

Balaban et al., $2005^{18}$ demonstrated that pulmonary function as measured by FVC decreased with age to a greater extent in the non-treatment group than in either the deflazacort or prednisone group, a result that was significant between treated and untreated populations ( $p=0.020$ to 0.046 ) but not between the two treatment options ( $p=0.593)$. Over 65 months of treatment, Schara et al., $2001^{15}$ demonstrated vital capacity was significantly improved in deflazacort treated versus untreated patients.

\section{Mortality}

Although cardiac care and pulmonary care have made the largest impact on prolonging survival in DMD patients, there is data to support the benefits of deflazacort on prolonging survival as well. Lebel et al., 201324 reporting results of a non-randomized comparative study which examined the long-term effect of deflazacort treatment on the development of scoliosis in 54 boys with DMD, found that after a 15-year period, five patients (21\%) in the non-treatment group had died versus one (3\%) in the deflazacort treatment group ( $p<0.005$, chi-square test). Biggar et al., $2006^{14}$, analyzing deflazacort treatment in 40 boys in their second decade compared to data from untreated boys over a 14 year period observed that only two of 40 (5\%) deflazacort-treated boys died at 13 and 18 years, whereas 12 of 34 (35\%) untreated boys died of cardiorespiratory complications in their second decade.

\section{Conclusion}

There are many reasons for lack of adherence to the DMD care guidelines, including access to care and the availability of specialized neuromuscular clinics across the US and internationally. Despite evidence on the benefits of glucocorticoids in DMD, MD STARnet reported that $54 \%$ of eligible patients are not treated with steroids. ${ }^{27}$ Specialized centers of excellence should directly enhance compliance to care guidelines. However, further education on the benefits of glucocorticoids is needed.

There are many DMD drugs currently in clinical trials and some even under review by the FDA. As new treatments become available, more centers of excellence are needed to standardize DMD care. The only medications available today for the treatment of DMD are corticosteroids. Prednisone is easily available and deflazacort is being imported from outside the United States. However, deflazacort may potentially be available in early 2017 and it has safety and efficacy advantages over prednisone. Although, there is an abundance of data demonstrating the benefits of deflazacort in both ambulatory and non-ambulatory DMD patients, more well-controlled studies are needed and currently are being planned to further assess its benefits in both younger DMD boys and non-ambulatory patients.

With the approval of new drugs in DMD, including both steroids and non-steroids, coordination of care and education are imperative to ensure that all eligible patients are informed and have access to new treatments. Because DMD care is seldom exclusive to a single center of excellence the resulting effect may be suboptimal. With the tremendous increase in DMD research, academia, advocacy groups and the pharmaceutical industry must work together to ensure all DMD patients are treated optimally and have easy access to comprehensive care and new treatments.
1. Mendell JR, Shilling C, Leslie ND, et al., Evidence-based path to newborn screening for Duchenne muscular dystrophy, Ann Neurol, 2012:71(3):304-313.

2. Koenig M, Hoffman EP, Bertelson CJ, et al., Complete cloning of the Duchenne Muscular dystrophy (DMD) CDNA and preliminary genomic organization of the DMD gene in normal and affected individuals, Cell, 1987;50(3):509-517.

3. Ciafaloni E, Fox DJ, Pandya S, et al., Delayed diagnosis in Duchenne muscular dystrophy: data from the Muscular Dystrophy Survellaince Track and Research Network (MD STARnet), J Pediat, , 2009:155(3):380-385

4. Humbertclaude V, Hamroun D, Bezzou K, et al., Motor and respiratory heterogeneity in Duchenne patients: implication for clinical trials, Eur J Paediatr Neurol, 2012;16(2):149-160

5. Eagle M, Baudouin S, Chandler C, et al., Survival in Duchenne muscular dystrophy: improvements in life expectancy since 1967 and the impact of home nocturnal ventilation, Neuromuscul Disord, 2002:12(10):926-929.

6. Bushby $K$, Finkel R, Birnkrant DJ, et al., for the DMD Care Considerations Working Group. Diagnosis and management of Duchenne muscular dystrophy, part 1: diagnosis, and pharmacological and psychosocial management, Lancet Neuro. 2010a; $9(1): 77-93$

7. Bushby K, Finkel R, Birnkrant DJ, et al., for the DMD Care Considerations Working Group. Diagnosis and management of Duchenne muscular dystrophy part 2: implementation of multidisciplinary care Lancet Neurol, 2010b:9(2):177-189.

8. Moxley RT III. Ashwal S, Pandya S, et al., Practice parameter: corticosteroid treatment of Duchenne dystrophy: report of the quality standards subcommittee of the American Academy of Neurology and the practice committee of the Child Neurology Society, Neurology, 2005;64(1):13-20.
9. Kinnett K, Rodger S, Vroom E, et al., Imperatives for DUCHENNE MD: a Simplified Guide to Comprehensive Care for Duchenne Muscular Dystrophy PLOS Curr, 2015:7:ecurrents.md.87770501e86 f36f1c71e0a5882edgba1.

10. Bladen CL, Rafferty K, Straub V, et al., The TREAT-NMD Duchenne muscular dystrophy registries: conception, design, and utilization by industry and academia, Hum Mutat, 2013;34(11):1449-57.

11. Griggs, RC, Bushby K. Finding the Optimum Regimen for Duchenne Muscular Dystrophy (FOR-DMD). In: ClinicalTrials.gov [Internet]. Bethesda (MD): National Library of Medicine (US). 2000-[2016 Jan 26] Available from: htps//clinicaltrialsgov/ct2/show/2016 NCT01603407?term = for-dmd\&rank=1: NCT01603407. (accessed: NCT01603407?term

12. Biggar WD, Gingras $M$, Fehlings $D L$, et al., Deflazacort treatment of Duchenne muscular dystrophy, J Pediatr, 2001;138(1):45-50.

13. Alman BA, Raza SN, Biggar WD, Steroid treatment and the 
Neuromuscular Disorders Duchenne Muscular Dystrophy

development of scoliosis in males with duchenne muscular dystrophy, J Bone Joint Surg Am, 2004;86-A(3):519-24.

14. Biggar WD, Harris VA, Eliasoph L, Alman B, Long-term benefits of deflazacort treatment for boys with Duchenne muscular dystrophy in their second decade, Neuromuscul Disord, 2006;16(4):249-55.

15. Schara U, Mortier, Mortier W, Long-Term Steroid Therapy in Duchenne Muscular Dystrophy-Positive Results versus Side Effects, J Clin Neuromuscul Dis, 2001;2(4):179-83.

16. Brooke $\mathrm{MH}, \mathrm{A}$ randomized trial of deflazacort and prednisone in Duchenne muscular dystrophy: efficacy and toxicity [abstract], Neurology, 1996;46:A476.

17. Bonifati MD, Ruzza $\mathrm{G}$, Bonometto P, et al., A multicenter, doubleblind, randomized trial of deflazacort versus prednisone in Duchenne muscular dystrophy, Muscle Nerve, 2000;23(9):1344-7.

18. Balaban B, Matthews DJ, Clayton GH, Carry T, Corticosteroid treatment and functional improvement in Duchenne muscular dystrophy: Iong-term effect, Am J Phys Med Rehabil, 2005;84(11):843-50

19. Angelini $C$, Pegoraro $E$, Turella $E$, et al., Deflazacort in Duchenne dystrophy: study of long-term effect, Muscle Nerve, 1994;17(4):386-391.

20. Houde S, Filiatrault M, Fournier A, Deflazacort use in Duchenne muscular dystrophy: an 8-year follow-up, Pediatr Neurol, 2008;38(3):200-6.

21. Biggar WD, Politano L, Harris VA, et al., Deflazacort in Duchenne muscular dystrophy: a comparison of two different protocols, Neuromuscul Disord, 2004:14(8-9):476-82.

22. Wang RT, Silverstein Fadlon CA, UIm JW, Online self-report data for duchenne muscular dystrophy confirms natural history and can be used to assess for therapeutic benefits, PLOS curr, 2014;17;6

23. Bello L, Gordish-Dressman H, Morgenroth LP, et al., Prednisone/ prednisolone and deflazacort regimens in the CINRG Duchenne Natural History Study, Neurology, 2015;85(12):1048-55.

24. Lebel DE, Corston JA, MCAdam LC, et al., Glucocorticoid treatment for the prevention of scoliosis in children with Duchenne muscular dystrophy: long-term follow-up, J Bone Joint Surg Am, 2013;95(12):1057-61.

25. Mavrogeni S, Papavasiliou A, Douskou M, et al., Effect of deflazacort on cardiac and sternocleidomastoid muscles in Duchenne muscular dystrophy: a magnetic resonance imaging study, Eur J Paediatr Neurol, 2009;13(1):34-40.

26. Silversides CK, Webb GD, Harris VA, Biggar DW, Effects of deflazacort on left ventricular function in patients with Duchenne muscular dystrophy, Am J Cardiol, 2003;91(6):769-72.

27. Kim S, Campbell KA, Fox DJ, et al., Corticosteroid treatments in males with Duchenne muscular dystrophy: treatment duration an time to loss of ambulation, J Child Neurol, 2015;30(10):1275-1280. 